\title{
Erratum to: Frequency response of primary resonance of electrostatically actuated CNT cantilevers
}

\author{
Dumitru I. Caruntu • Le Luo
}

Published online: 28 August 2014

(C) Springer Science+Business Media Dordrecht 2014

\section{Erratum to: Nonlinear Dyn \\ DOI: 10.1007/s11071-014-1537-4}

1. Equation (16) should be corrected as

$$
\begin{gathered}
\varepsilon^{1}: D_{0}^{2} w_{1}+\frac{\partial^{4} w_{1}}{\partial z^{4}}=-2 D_{0} D_{1} w_{0}-b^{*} D_{0} w_{0} \\
+\delta \sum_{k=0}^{3} \alpha_{k} w_{0}^{k} \cos ^{2} \Omega^{*} T_{0}+\mu \sum_{k=0}^{3} \lambda_{k} w_{0}^{k}
\end{gathered}
$$

$$
\begin{aligned}
& \left.\left.+3 A^{2} \bar{A} e^{i \omega_{k} T_{0}}+3 \bar{A}^{2} A e^{-i \omega_{k} T_{0}}+\bar{A}^{3} e^{-3 i \omega_{k} T_{0}}\right)\right] \\
& +\mu\left[\lambda_{0}+\lambda_{1} \phi_{k}\left(A e^{i \omega_{k} T_{0}}+\bar{A} e^{-i \omega_{k} T_{0}}\right)\right. \\
& +\lambda_{2} \phi_{k}^{2}\left(A^{2} e^{2 i \omega_{k} T_{0}}+2 \bar{A} A+\bar{A}^{2} e^{-2 i \omega_{k} T_{0}}\right) \\
& +\lambda_{3} \phi_{k}^{3}\left(A^{3} e^{3 i \omega_{k} T_{0}}\right. \\
& \left.\left.+3 A^{2} \bar{A} e^{i \omega_{k} T_{0}}+3 \bar{A}^{2} A e^{-i \omega_{k} T_{0}}+\bar{A}^{3} e^{-3 i \omega_{k} T_{0}}\right)\right]
\end{aligned}
$$

3. Equation (20) should be corrected as

$$
\begin{aligned}
& D_{0}^{2} w_{1}+\frac{\partial^{4} w_{1}}{\partial z^{4}}=-2 i \omega_{k}\left(A^{\prime} e^{i \omega_{k} T_{0}}-\bar{A}^{\prime} e^{-i \omega_{k} T_{0}}\right) \phi_{k} \\
& -i b^{*} \omega_{k}\left(A e^{i \omega_{k} T_{0}}-\bar{A} e^{-i \omega_{k} T_{0}}\right) \phi_{k} \\
& +\frac{\delta}{4}\left(e^{2 i \Omega^{*} T_{0}}+2+e^{-2 i \Omega^{*} T_{0}}\right) \\
& {\left[\alpha_{0}+\alpha_{1} \phi_{k}\left(A e^{i \omega_{k} T_{0}}+\bar{A} e^{-i \omega_{k} T_{0}}\right)\right.} \\
& +\alpha_{2} \phi_{k}^{2}\left(A^{2} e^{2 i \omega_{k} T_{0}}+2 \bar{A} A+\bar{A}^{2} e^{-2 i \omega_{k} T_{0}}\right) \\
& +\alpha_{3} \phi_{k}^{3}\left(A^{3} e^{3 i \omega_{k} T_{0}}\right.
\end{aligned}
$$

The online version of the original article can be found under doi:10.1007/s11071-014-1537-4.

\section{I. Caruntu $(\varangle) \cdot$ L. Luo}

Mechanical Engineering Department, University of Texas-Pan American, Edinburg, TX 78539, USA e-mail: caruntud@utpa.edu; caruntud2@asme.org; dcaruntu@yahoo.com

$$
\begin{aligned}
& -2 i \omega_{k} g_{1 k k}\left(A^{\prime} e^{i \omega_{k} T_{0}}-\bar{A}^{\prime} e^{-i \omega_{k} T_{0}}\right) \\
& -i b^{*} \omega_{k} g_{1 k k}\left(A e^{i \omega_{k} T_{0}}-\bar{A} e^{-i \omega_{k} T_{0}}\right) \\
& +\frac{\delta}{4}\left(e^{2 i \Omega^{*} T_{0}}+e^{-2 i \Omega^{*} T_{0}}+2\right) \\
& {\left[\alpha_{0} g_{0 k k}+\alpha_{1} g_{1 k k}\left(A e^{i \omega_{k} T_{0}}+\bar{A} e^{-i \omega_{k} T_{0}}\right)\right.} \\
& +\alpha_{2} g_{2 k k}\left(A^{2} e^{2 i \omega_{k} T_{0}}+2 A \bar{A}+\bar{A}^{2} e^{-2 i \omega_{k} T_{0}}\right) \\
& +\alpha_{3} g_{3 k k}\left(A^{3} e^{3 i \omega_{k} T_{0}}\right. \\
& \left.\left.+3 A^{2} \bar{A} e^{i \omega_{k} T_{0}}+3 A \bar{A}^{2} e^{-i \omega_{k} T_{0}}+\bar{A}^{3} e^{-3 i \omega_{k} T_{0}}\right)\right] \\
& +\mu\left[\lambda_{0} g_{0 k k}+\lambda_{1} g_{1 k k}\left(A e^{i \omega_{k} T_{0}}+\bar{A}^{-i \omega_{k} T_{0}}\right)\right. \\
& +\lambda_{2} g_{2 k k}\left(A^{2} e^{2 i \omega_{k} T_{0}}+2 A \bar{A}+\bar{A}^{2} e^{-2 i \omega_{k} T_{0}}\right) \\
& +\lambda_{3} g_{3 k k}\left(A^{3} e^{3 i \omega_{k} T_{0}}+3 A^{2} \bar{A} e^{i \omega_{k} T_{0}}\right. \\
& \left.\left.+3 \bar{A}^{2} e^{-i \omega_{k} T_{0}}+\bar{A}^{3} e^{-3 i \omega_{k} T_{0}}\right)\right]=0
\end{aligned}
$$


4. Equation (22) should be corrected as

$-2 i \omega_{k} g_{1 k k} A^{\prime}-i b^{*} \omega_{k} g_{1 k k} A$

$+\frac{\delta}{4} \cdot 2\left[\alpha_{1} g_{1 k k} A+3 \alpha_{3} g_{3 k k} A^{2} \bar{A}\right]$

$+\mu\left[\lambda_{1} g_{1 k k} A+3 \lambda_{3} g_{3 k k} A^{2} \bar{A}\right]$

+ "other secular terms" $=0$

5. After Eq. (22) the text should read:

The given terms in Eq. (22) come from all terms in Eq. (25) except the ones multiplied by $e^{2 i \Omega^{*} T_{0}}+$ $e^{-2 i \Omega^{*} T_{0}}$. The "other secular terms" come from multiplying the first bracketed expression of Eq. (19) by $e^{2 i \Omega^{*} T_{0}}+e^{-2 i \Omega^{*} T_{0}}$.

6. Equation (23) should be corrected

$$
\begin{aligned}
& \frac{\delta}{4}\left\{\alpha_{0} g_{0 k k}\left(e^{2 i \Omega^{*} T_{0}}+e^{-2 i \Omega^{*} T_{0}}\right)\right. \\
& +\alpha_{1} g_{1 k k}\left(A e^{i\left(\omega_{k}+2 \Omega^{*}\right) T_{0}}\right. \\
& +A e^{i\left(\omega_{k}-2 \Omega^{*}\right) T_{0}}+\bar{A} e^{-i\left(\omega_{k}-2 \Omega^{*}\right) T_{0}} \\
& \left.+\bar{A} e^{-i\left(\omega_{k}+2 \Omega^{*}\right) T_{0}}\right) \\
& +\alpha_{2} g_{2 k k}\left(A^{2} e^{2 i\left(\omega_{k}+\Omega^{*}\right) T_{0}}+2 A \bar{A} e^{2 i \Omega^{*} T_{0}}\right. \\
& +\bar{A}^{2} e^{-2 i\left(\omega_{k}-\Omega^{*}\right) T_{0}} \\
& +A^{2} e^{2 i\left(\omega_{k}-\Omega^{*}\right) T_{0}}+2 A \bar{A} e^{-2 i \Omega^{*} T_{0}} \\
& \left.+\bar{A}^{2} e^{-2 i\left(\omega_{k}+\Omega^{*}\right) T_{0}}\right) \\
& +\alpha_{3} g_{3 k k}\left[A^{3} e^{i\left(3 \omega_{k}+2 \Omega^{*}\right) T_{0}}\right. \\
& +3 A^{2} \bar{A} e^{i\left(\omega_{k}+2 \Omega^{*}\right) T_{0}}+3 A \bar{A}^{2} e^{-i\left(\omega_{k}-2 \Omega^{*}\right) T_{0}} \\
& +\bar{A}^{3} e^{-i\left(3 \omega_{k}-2 \Omega^{*}\right) T_{0}}+A^{3} e^{i\left(3 \omega_{k}-2 \Omega^{*}\right) T_{0}} \\
& +3 A^{2} \bar{A} e^{i\left(\omega_{k}-2 \Omega^{*}\right) T_{0}}+3 A \bar{A}^{2} e^{-i\left(\omega_{k}+2 \Omega^{*}\right) T_{0}} \\
& \left.\left.+\bar{A}^{3} e^{-i\left(3 \omega_{k}+2 \Omega^{*}\right) T_{0}}\right]\right\}=0
\end{aligned}
$$

7. The first two sentences to include Eq. (24) in Sect. 5 should be corrected as

The frequency of AC actuation is near half natural frequency $\Omega^{*} \cong \omega_{k} / 2$. This can be written as

$\Omega^{*}=\frac{\omega_{k}}{2}+\varepsilon \sigma$

where $\sigma$ is a detuning parameter and $\Omega^{*} T_{0}=$ $\omega_{k} T_{0} / 2+\sigma T_{1}$.
8. Equation (28) should be corrected as

$$
\begin{aligned}
& -2 i \omega_{k}\left(\frac{1}{2} a^{\prime} e^{i \beta}+i \frac{1}{2} a \beta^{\prime} e^{i \beta}\right) g_{1 k k} \\
& -i b^{*} \omega_{k} \frac{1}{2} a e^{i \beta} g_{1 k k} \\
& +\frac{\delta}{2}\left(\alpha_{1} \frac{1}{2} a e^{i \beta} g_{1 k k}+\alpha_{3} g_{3 k k} \frac{3}{8} a^{3} e^{i \beta}\right) \\
& +\mu\left(\lambda_{1} \frac{1}{2} a e^{i \beta} g_{1 k k}+\lambda_{3} g_{3 k k} \frac{3}{8} a^{3} e^{i \beta}\right) \\
& +\frac{\delta}{4}\left(\alpha_{2} g_{2 k k} \frac{1}{4} a^{2}\right) e^{2 i \beta-2 i \sigma T_{1}} \\
& +\frac{\delta}{4}\left(\alpha_{0} g_{0 k k}+\alpha_{2} g_{2 k k} \frac{1}{2} a^{2}\right) e^{2 i \sigma T_{1}}=0
\end{aligned}
$$

9. Equation (29) should be corrected as

$$
\begin{aligned}
& -i \omega_{k}\left(a^{\prime}+a i \beta^{\prime}\right) g_{1 k k}-i b^{*} \omega_{k} \frac{1}{2} a g_{1 k k} \\
& +\frac{\delta}{2}\left(\alpha_{1} \frac{1}{2} a g_{1 k k}+\alpha_{3} g_{3 k k} \frac{3}{8} a^{3}\right) \\
& +\mu\left(\lambda_{1} \frac{1}{2} a g_{1 k k}+\lambda_{3} g_{3 k k} \frac{3}{8} a^{3}\right) \\
& +\frac{\delta}{16}\left(\alpha_{2} g_{2 k k} a^{2}\right) e^{i \beta-2 i \sigma T_{1}} \\
& +\frac{\delta}{4}\left(\alpha_{0} g_{0 k k}+\alpha_{2} g_{2 k k} \frac{1}{2} a^{2}\right) e^{2 i \sigma T_{1}-i \beta}=0
\end{aligned}
$$

10. Equation (30) should be corrected as

$$
\gamma=2 \sigma T_{1}-\beta
$$

11. Equation (32) should be corrected as

$$
\begin{aligned}
& \gamma^{\prime}=2 \sigma+\frac{\delta}{\omega_{k}}\left(\alpha_{1} \frac{1}{4}+\alpha_{3} \frac{g_{3 k k}}{g_{1 k k}} \frac{3}{16} a^{2}\right) \\
& +\frac{\mu}{\omega_{k}}\left(\lambda_{1} \frac{1}{2}+\lambda_{3} \frac{g_{3 k k}}{g_{1 k k}} \frac{3}{8} a^{2}\right) \\
& +\frac{\delta}{\omega_{k}} \alpha_{2} \frac{g_{2 k k}}{g_{1 k k}} \frac{1}{16} a \cos \gamma \\
& +\frac{\delta}{\omega_{k}}\left(\frac{\alpha_{0}}{4} \frac{g_{0 k k}}{g_{1 k k} a}+\alpha_{2} \frac{g_{2 k k}}{g_{1 k k}} a \frac{1}{8}\right) \cos \gamma
\end{aligned}
$$


12. Equation (34) should be corrected as

$$
\begin{aligned}
\sigma= & -\frac{\delta}{\omega_{k}}\left(\alpha_{1} \frac{1}{8}+\alpha_{3} \frac{g_{3 k k}}{g_{1 k k}} \frac{3}{32} a^{2}\right) \\
& -\frac{\mu}{\omega_{k}}\left(\lambda_{1} \frac{1}{4}+\lambda_{3} \frac{g_{3 k k}}{g_{1 k k}} \frac{3}{16} a^{2}\right)
\end{aligned}
$$

$-\frac{\delta}{\omega_{k}} \alpha_{2} \frac{g_{2 k k}}{g_{1 k k}} \frac{1}{32} a \cos \gamma$

$-\frac{\delta}{\omega_{k}}\left(\frac{\alpha_{0}}{8} \frac{g_{0 k k}}{g_{1 k k} a}+\alpha_{2} \frac{g_{2 k k}}{g_{1 k k}} a \frac{1}{16}\right) \cos \gamma$ 